\title{
Ja zum Ausbau der Interprofessionalität - Nein zur Initiative
}

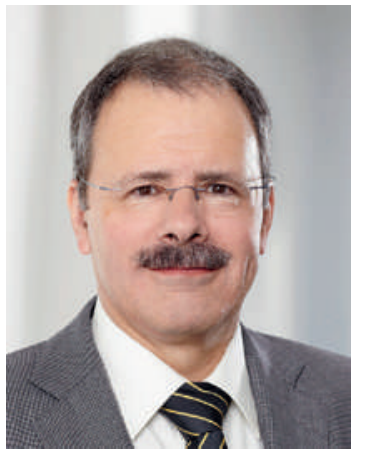

Die Parlamentarische Initiative «Gesetzliche Anerkennung der Verantwortung der Pflege» fordert, dass Pflegefachpersonen zukünftig einen Teil ihrer Leistungen in eigener Verantwortung ohne ärztliche Verordnung erbringen können. Welche Leistungen dies genau sind, blieb bislang undefiniert.

Über diese Kernforderung hinaus lenkt die Initiative den Blick auf die Zusammenarbeit der Gesundheitsberufe im Allgemeinen und wie diese zukünftig ausgebaut werden kann. Sie greift mit der Interprofessionalität im Gesundheitswesen ein auch in der Ärzteschaft als sehr wichtig erachtetes Thema auf.

In diesem Sinne spiegelt die interne Vernehmlassung der FMH zur Initiative nicht nur eine hohe Wertschätzung der Arbeit der Pflegefachpersonen wider, sondern auch eine grosse Offenheit gegenüber einer Erweiterung ihrer Kompetenzen. Gleichzeitig besteht jedoch weitgehend Konsens darüber, dass eine solche Neugestaltung der Interprofessionalität aus verschiedenen Gründen anders umgesetzt werden sollte, als es die Initiative skizziert.

Entscheidend dafür, dass die Weiterentwicklung des Pflegeberufs - und anderer Gesundheitsberufe - dem Patienten zum Vorteil und nicht zum Nachteil gereicht, ist nach Auffassung der FMH die Wahrung der Koordinations- und Entscheidfunktion des Arztes, insbesondere in Hinblick auf Diagnosestellung und Therapieentscheide.

Nur so kann einer Fragmentierung der Behandlung entgegengewirkt werden.

\section{Die Interprofessionalität ist zu fördern, sie sollte aber anders umgesetzt wer- den, als es die Initiative skizziert.}

Für die Patientenversorgung lässt die Initiative nicht nur offen, welche Leistungen konkret von den Pflegenden «in eigener Verantwortung erbracht werden» sollen - sondern auch, wer diese festlegt und fortan anpasst. Eine Abgrenzung ärztlicher und pflegerischer Kompetenzen durch Ausführungsbestimmungen der Verwaltung erscheint uns für den Behandlungsalltag nicht tragfähig. Gegen eine per Gesetz festgelegte Übertragung weitreichenderer Verantwortung an Pflegefachkräfte spricht weiterhin das inhomogene Ausbildungsniveau im Pflegebereich - von der Fachfrau Gesundheit über die Pflegefachfrau bis zum Master of Science in Pflege.

Für die Organisation des Behandlungsalltags - und damit für den Patienten - wäre es folglich am zuträglichsten, wenn Kompetenzerweiterungen der Pflege im Rahmen des bewährten Delegationsmodells umgesetzt würden. Dieses ermöglicht situativ und individuell angepasste Entscheidungen des delegierenden Arztes und wird nicht zuletzt auch denjenigen Pflegenden gerecht, die eine weiterreichende Verantwortung nur in abgegrenzten Bereichen übernehmen möchten oder können. Auch in Hinblick auf die andernfalls komplexen haftungsrechtlichen Fragen ist dieses Modell am geeignetsten ein Problem, das die Initiative nicht anspricht, dessen Klärung für die Arbeit am Patienten aber eine unverzichtbare Voraussetzung darstellt.

\section{Kompetenzerweiterungen sind zu unterstützen - Hand dazu bietet das bewährte Delegationsmodell.}

Die von den Initianten in Aussicht gestellte Kostensenkung durch den Verzicht auf die ärztliche Verschreibung erscheint unwahrscheinlich. Das Neuetablieren direkter Zugänge zu Gesundheitsleistungen ohne ärztliche Indikationskontrolle lässt eher einen Kostenanstieg durch Mengenausweitung befürchten.

Ziele wie Kompetenzerweiterungen der Pflege und eine Attraktivitätssteigerung des Pflegeberufs werden von der Ärzteschaft voll und ganz geteilt. Wir plädieren aber für andere Massnahmen zu ihrer Erreichung: Anstatt pauschal grössere Verantwortungsbereiche festzulegen, sollten die Möglichkeiten zur situationsadäquaten Delegation deutlich erweitert werden.

Die Interprofessionalität ist eine Herausforderung, die alle Gesundheitsberufe gemeinsam meistern müssen und in der es immer wieder Prozesse neu abzustimmen gilt. Angesichts der sehr guten Zusammenarbeit zwischen Pflegenden und Ärzten bin ich optimistisch, dass uns - trotz unserer Ablehnung der Initiative - gemeinsam der Ausbau der Interprofessionalität gelingen wird, zum Vorteil der Patienten.

Dr. med. Jürg Schlup, Präsident der FMH 